\title{
Propuesta de UNA ARQUiTeCTURA DE LA INDUSTRIA 4.0 EN LA CADENA DE SUMINISTRO DESDE LA PERSPECTIVA DE LA INGENIERÍA INDUSTRIAL
}

\author{
Mónica Johanna Blanco Rojas ${ }^{1}$, Karen Tatiana González Rojas², \\ José Ignacio Rodríguez Molano ${ }^{3}$ \\ ${ }^{1}$ Estudiante de Ingeniería Industrial \\ ${ }^{2}$ Estudiante de Ingeniería Industrial. Correo electrónico: ktgonzalezr@correo.udistrital.edu.co \\ ${ }^{3}$ Doctor en Ingeniería Informática. Profesor \\ Universidad Distrital Francisco José de Caldas, Bogotá, Colombia
}

Fecha de recibido: 10 de mayo del 2017

Fecha de aprobado: 23 de agosto del 2017

Cómo citar este artículo: M. J. Blanco-Rojas, K. T. González-Rojas y J. I. Rodríguez-Molano, "Propuesta de una arquitectura de la industria 4.0 en la cadena de suministro desde la perspectiva de la ingeniería industrial", Ingeniería Solidaria, vol. 13, n. ${ }^{\circ}$ 23, Sept. 2017, pp. 77-90. doi: https://doi.org/10.16925/in.v23i13.2007

Resumen: Introducción: este artículo es producto de la investigación sobre aplicaciones del big data, desarrollada en la Universidad Distrital Francisco José de Caldas en el 2017, la cual busca solucionar el problema de compatibilidad entre la información generada por sensores en equipos y máquinas con las plataformas que soportan la información. Se muestra una propuesta de arquitectura que pueden adoptar las cadenas de suministro inmersas en la industria 4.0. Se identificaron los elementos, autores y partes interesadas que interactúan en la arquitectura. Metodología: a) se argumenta la importancia de la industria 4.0; b) se expone que las organizaciones deben adaptarse a la nueva revolución industrial; c) se realiza la revisión de la literatura de investigaciones. A estos hallazgos se les realiza un análisis metódico de las propuestas, avances, metodología, investigaciones futuras, resultados y conclusiones. d) Se plantea una arquitectura; e) se crea una aplicación móvil para evaluar la interconexión de la capa de sensores con la capa de aplicación planteada en la arquitectura; f) se comprueba la usabilidad de la aplicación, y g) se exponen las ventajas y limitaciones de la arquitectura. Resultados: la aplicación tiene una usabilidad del 91\%, permitiendo que haya conexión en tiempo real entre la capa de sensores con la capa de aplicación. Conclusiones: la presente investigación expone herramientas que brindan a la cadena de suministro directrices para incluirse en la industria 4.0 y obtener ventajas competitivas.

Palabras clave: aplicación móvil, arquitectura, big data, industria 4.0, Internet de las cosas. 


\title{
Proposed INDUSTRY 4.0 ARCHITECTURE IN THE SUPPLY CHAIN FROM THE PERSPECTIVE OF INDUSTRIAL ENGINEERING
}

\begin{abstract}
Introduction: This article derives from the research into big data applications, conducted at the Universidad Distrital Francisco José de Caldas in 2017, which seeks to solve the problem of compatibility between the information generated by sensors in equipment and machines, and the platforms that support such information. A proposed architecture that can be adopted by supply chains immersed in Industry 4.0 is shown. The elements, authors, and stakeholders that interact in the architecture were identified. Methodology: a) the importance of Industry 4.0 is argued; b) it is stated that organizations must adapt to the new industrial revolution; c) the research literature is reviewed. These findings are methodically analyzed for proposals, advances, methodology, future research, results and conclusions; d) an architecture is proposed; e) a mobile application is created to evaluate the interconnection of the sensor layer with the application layer proposed by the architecture; $f$ ) the usability of the application is checked; and g) the advantages and limitations of the architecture are explained. Results: The application has a $91 \%$ usability allowing real-time connection between the sensor layer and the application layer. Conclusions: This research presents tools that provide the supply chain with guidelines to be included in Industry 4.0 and to obtain competitive advantages.
\end{abstract}

Keywords: mobile application, architecture, big data, Industry 4.0, Internet of things.

\section{Proposta de UMA ARQuitetura da INDÚstria}

\section{O NA REDE DE FORNECIMENTO DESDE A PERSPECTIVA DA ENGENHARIA INDUSTRIAL}

Resumo. Introdução: este artigo é produto da pesquisa sobre aplicativos de big data, desenvolvida na Universidade Distrital Francisco José de Caldas em 2017, na qual busca-se solucionar o problema de compatibilidade entre a informação gerada por sensores em equipamentos e máquinas com as plataformas que suportam a informação. Apresenta-se uma proposta de arquitetura que as redes de fornecimento imersas na indústria 4.0 podem adotar. Foram identificados os elementos, autores e partes interessadas que interagem na arquitetura. Metodologia: a) argumenta-se sobre a importância da indústria 4.0; b) expõe-se que as organizações devem se adaptar à nova revolução industrial; c) realiza-se a revisão da literatura de investigações. Essas descobertas passam por uma análise metódica das propostas, avanços, metodologia, futuras pesquisas, resultados e conclusões; d) uma arquitetura é apresentada; e) um aplicativo móvel é criado para avaliar a interconexão da camada de sensores com a camada de aplicativo projetada na arquitetura; f) a usabilidade do aplicativo é comprovada, e g) as vantagens e limitações da arquitetura são expostas. Resultados: o aplicativo tem uma usabilidade de $91 \%$, o que permite que haja conexão em tempo real entre a camada de sensores e a camada de aplicativo. Conclusões: a presente pesquisa expõe ferramentas que propiciam diretrizes à rede de fornecimento para serem incluídas na indústria 4.0 e obter vantagens competitivas.

Palavras-chave: aplicativo móvel, arquitetura, big data, indústria 4.0, internet das coisas. 


\section{Introducción}

La industria 4.0 (I4) se originó en un proyecto de estrategia de alta tecnología del gobierno alemán en el 2011. Con frecuencia se refiere como la cuarta revolución industrial. I4 permite un cambio de paradigma de la producción "centralizada" a la "descentralizada" [1], lo que genera una estrategia para ser competitivo en el futuro [2], en la cual los productos tienden a controlar su propio procesamiento de fabricación [3]. Adicionalmente, se centra en la optimización de las cadenas de valor mediante la producción dinámica y autónomamente controlada, lo que automatiza las industrias mediante el intercambio de datos entre los eslabones de la cadena de suministro [4].

Una parte integral de la fábrica del futuro es la extensión y expansión de las cadenas de suministro; esto rompe las barreras comerciales tradicionales y trasciende las cuatro paredes de una sola instalación para trabajar cada vez más estrechamente con clientes, proveedores, organismos industriales y el mundo académico. Esta idea de la empresa extendida es fundamental para todo el concepto de "I4", en la cual esta forma colaborativa de trabajo es vista como la una manera de optimizar la productividad [5].

En este sentido, la I4 está basada principalmente en el Internet de las cosas (Іот), big data y la fabricación inteligente [6], en donde los productos en proceso, los componentes y las máquinas de producción recogen y comparten datos en tiempo real [7] que pueden utilizarse en la predicción de fallas [8], en la mejora de la fabricación [3], y en la toma de decisiones descubriendo debilidades y teniendo en cuenta la situación actual del sistema [9]. La I4 junto con IOT puede hacer una gran revolución en la gestión de la cadena de suministro global [4] al permitir alcanzar niveles sin precedentes de eficiencia operativa y acelerar el crecimiento de la productividad [10], [11].

Los datos juegan un papel importante en las diferentes decisiones relacionadas con la cadena de suministro de la empresa; estos son voluminosos, versátiles, rápidos y sensibles, que son características del big data [4], [12]. El éxito de cualquier negocio se basa en laeficiencia de su cadena de suministro, que es responsable de crear y mantener los vínculos de diferentes entidades en un negocio; estas son responsables de la adquisición de materias primas para la entrega final del producto [13].
Las empresas están generando todo el tiempo información a partir de sensores y de los equipos que se usan en la producción; sin embargo, no suelen ser compatibles en la manera en que generan la información ni en el orden de esta. Con el diseño de la arquitectura, se busca que la información recolectada por los eslabones de la cadena de suministro sea estandarizada y se pueda usar de manera fácil y en tiempo real en cualquier punto de la misma.

El resto de este trabajo está estructurado de la siguiente manera: en la sección 2 presentamos los antecedentes y revisión bibliográfica sobre los temas emergentes de la I4; en la sección 3, explicamos la metodología usada para la elaboración del artículo; y la sección 4 presenta la propuesta de la arquitectura para la cadena de suministro en el contexto de la I4. Se continúa con la sección 5 , en la cual se evalúa la conexión entre las capas de usuario y la aplicación por medio de la aplicación móvil "ERP for I4". Los resultados y discusión se exponen en la sección 6; y se finaliza con las secciones 7, en la cual se presentan las conclusiones, y 8 , que contiene las futuras investigaciones.

\subsection{Antecedentes de investigación}

El IOT es un tema emergente de importancia técnica, social y económica, además de ser un paradigma que permite la interconexión de dispositivos para la adquisición de datos [14], comunicación y colaboración entre ellos para alcanzar objetivos comunes [15]-[16], [17], así como la optimización de recursos y reducción de costos [18]. El IOT permite a las empresas modernas adoptar nuevas estrategias basadas en datos y manejar la presión competitiva global más fácilmente.

Sin embargo, la adopción de гот incrementa el volumen total de los datos generados transformando los datos en big data [19], para lo cual se necesitan tecnologías y sistemas diseñados para recopilar, descubrir y analizar de manera efectiva diferentes tipos de grandes datos y extraer un valor para la organización [20], utilizando diferentes herramientas, tales como sistemas de negocios inteligentes, minería de datos yanálisis predictivo [13]. Una ventaja del big data es que puede ignorar las restricciones físicas y geográficas; realizar la conectividad y la descentralización de los recursos de información, y resolver el problema de la "isla aislada de la información" [20], lo que dismi- 
nuye las brechas entre los eslabones de la cadena de suministro.

La transformación de las $5 \mathrm{~V}$ de big data es un gran desafío para la capacidad de procesamiento [21]. La computación en la nube (CC) ha surgido como un nuevo paradigma para proporcionar la computación como un servicio de utilidad para abordar las diferentes necesidades de procesamiento [22]. CC integra tecnologías o arquitecturas tales como el гот y el big data para lograr una meta, ofrecer una plataforma o construir una solución [23]; cada vez más compañías lo emplean, ya que proporciona flexibilidad y agilidad para apoyar las operaciones de la cadena de suministro [24]. En la computación en la nube, la infraestructura de hardware no se ve, pero lo que el usuario o los clientes ven es la interfaz de aplicación; debido al uso de Internet como unidad de transporte o factor de enlace entre aplicaciones y hardware, se puede acceder a la computación en la nube en cualquier momento o lugar donde haya una conexión a Internet disponible [25].

Así mismo, los proveedores de CC han sido valiosas alternativas con el objetivo de acelerar las plataformas de aprendizaje de las máquinas. El aprendizaje automático $(\mathrm{ML})$ realiza tareas que necesitan mucho tiempo de ejecución y requieren plataformas capaces de disminuir estos tiempos [26]. Así mismo, realiza una comprensión profunda de patrones [27] en los datos, lo cual ayuda a tomar decisiones óptimas y eficientes, y mejora el rendimiento a través de la experiencia [28]. El objetivo principal del ML es crear un sistema capaz de dar una solución óptima cuando se le introduce información [29].

En resumen, en el aprendizaje automático, primero, el algoritmo analiza un conjunto de datos de "entrenamiento" para establecer una función capaz de distinguir sujetos individuales entre grupos. Una vez hecho esto, el modelo puede ser aplicado a un nuevo conjunto de datos, y la precisión del método se puede medir en este nuevo escenario [30]. El éxito del aprendizaje automático en tareas de inteligencia se debe, en gran medida, a su capacidad para descubrir una estructura compleja que no se especificó de antemano [31].

La arquitectura propuesta es la integración de la revisión de la literatura académica estudiada junto con los estudios que se describen a continuación, que cumplen con el modelo de arquitectura propuesta, junto con lo cual se realizó el ciclo de interacción de actores y elementos, y la adaptación de la arquitectura a la cadena de suministro con el planteamiento de sus componentes.

Se tomó como base la tesis doctoral "Metamodelo para la integración de la Internet de las cosas y redes sociales" [32]. Esta arquitectura general tiene tres niveles: en el primero se encuentran los sensores, los cuales obtienen información en tiempo real; la capa intermedia, denominada de comunicación, integra redes cableadas e inalámbricas que transfieren la información; y por último, está la capa superior, donde se encuentran las aplicaciones y que a su vez consta de tres capas: capa de tecnología, capa de middleware y la capa de aplicación. Esta arquitectura general se adecuó a la cadena de suministro inmersa en la I4.

Otro estudio que se tomó como referencia para realizar la propuesta de arquitectura es "Architecture for Industry 4.0-based Manufacturing Systems" [33], del cual se analizaron las capas de interfaz, procesamiento de información e inteligencia, y que sirvió como parámetros para los elementos que se utilizaron en esta investigación.

Por último, se usó el texto "An Architecture for Organizing and Locating Services to the Industry 4.0" [34], que propone una la visión global de la I4, en la cual los sistemas físicos se asignan a un mundo virtual: de aquí se tomó la base para crear el ciclo de interacción de actores y elemento de la arquitectura propuesta.

\section{Metodología}

Para cumplir con el objetivo de la investigación, se estudió la información disponible en bases de datos académicas realizando una revisión de estado de literatura de investigaciones. Un 15\% de publicaciones están comprendidas entre el 2012 y el 2014, y un 85\% en el periodo 2015-2017. La propuesta tiene este orden: primero, se argumenta por la importancia de la I4 presentando una introducción de esta junto con una conceptualización sobre sus elementos, como lo son el Internet de las cosas, big data, la computación en la nube, el aprendizaje automático y la cadena de suministro.

La siguiente sección muestra una propuesta de arquitectura para la cadena de suministro realizada a partir de un prototipo ya establecido para resolver problemas de integración de tecnologías e informa- 
ción para el desarrollo de aplicaciones en la I4, en la cual se establecieron y explicaron de manera explícita los elementos, autores y partes interesadas que componen dicha arquitectura.

Posteriormente, en los resultados, se presenta la arquitectura de la I4 dentro de la cadena de suministro, se validó la integración de la capa de sensores con la capa de aplicación mediante el desarrollo de la aplicación móvil ERP for I4. Se realizó esta validación con un modelo matemático que evalúa la usabilidad de la $a p p$ por medio de encuestas a expertos de la cadena de suministro y a usuarios que la usaron; luego, se abordan las discusiones de la arquitectura; por último, se establecen las observaciones finales y futuras investigaciones.

\section{Resultados}

\subsection{Propuesta de arquitectura}

De acuerdo con los artículos estudiados, se puede señalar que la 44 tiene cuatro pilares, que son la interconexión, la transparencia de la información, las decisiones descentralizadas y la asistencia técnica [35]; adicionalmente, se busca que la fabricación sea inteligente, es decir, que sea autoconsciente, autooptimizada y autoconfigurada para generar beneficios económicos en las compañías que lo empleen [3]. Cuando se implementa con éxito la I4 en la cadena de suministro, puede transformar la producción tradicional de células dispersas y aisladas a un flujo de producción totalmente integrado, automatizado y optimizado para lograr mayores eficiencias y relaciones de fabricación más cercanas entre los proveedores, productores y clientes [36].

En la figura 1 se han identificado los actores y elementos que interactúan en la cadena de suministro en el ambiente de la I4, lo que permitió establecer un marco general que recopiló la información en los enlaces que van a generar la arquitectura propuesta y así determinar los diferentes actores (empresa, negocios, demanda y aprendizaje automático entre otros) que realizan las interacciones para crear una cadena de suministro inteligente.

Al generarse una cadena de suministro inteligente, lo importante es el flujo de información, que empieza con la obtención de datos a través de la aplicación de Internet de las cosas en todos los

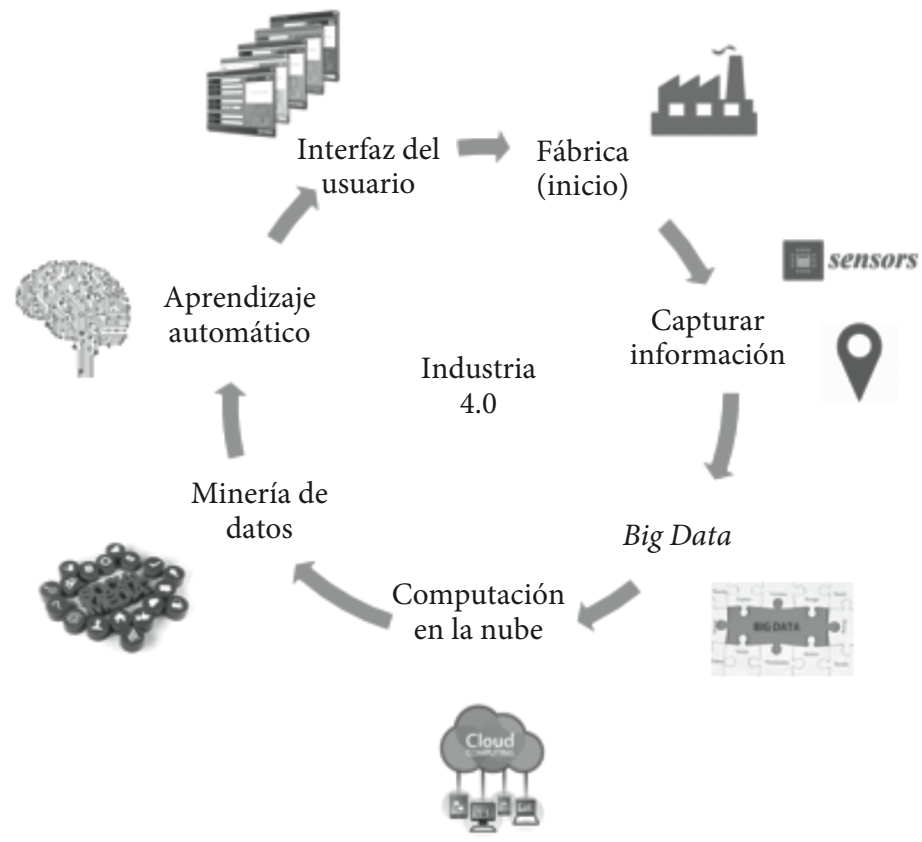

Figura 1. Ciclo de interacción de actores y elemento de la arquitectura propuesta Fuente: elaboración propia 
eslabones de la cadena de suministro, pasa por la limpieza y selección de datos en los procesos de big data, y posteriormente envía esta información a la computación en la nube, en la cual el aprendizaje automático brinda las soluciones óptimas. Luego de esto se alimenta la cadena de suministro enviando la información transformada a cada sección y tal como predicciones de oferta y demanda, planeaciones de rutas de distribución, pronósticos de fallas de maquinaria, planeación óptima de la producción, ventas asertivas satisfaciendo las necesidades de los clientes, entre otros.

La figura 2 muestra una arquitectura propuesta para la cadena de suministro en el contexto de la I4, en la cual se definen tres capas que buscan la integración de los diferentes elementos y actores.

A continuación, se describen los componentes de la arquitectura dentro de la cadena de suminis- tro en la i4, con el fin de establecer una propuesta integral que permita cubrir las necesidades de las organizaciones a la hora de formar parte de la 14.

\subsection{Capa de sensores}

Esta capa es la encargada de la recolección de datos en la cadena de suministro a través de diferentes medios, entre estos los sensores (que están en los diferentes agentes de la planta, como máquinas, locaciones y personal operativo), el componente de negocios (cada eslabón de la cadena de suministro genera información en tiempo real), las redes sociales (en las cuales se generará la comunicación y el cruce de información entre los clientes, proveedores y la organización), y, por último, los elementos de georreferenciación (que se encargan de la ubicación y recolección de datos en los canales de distribución).

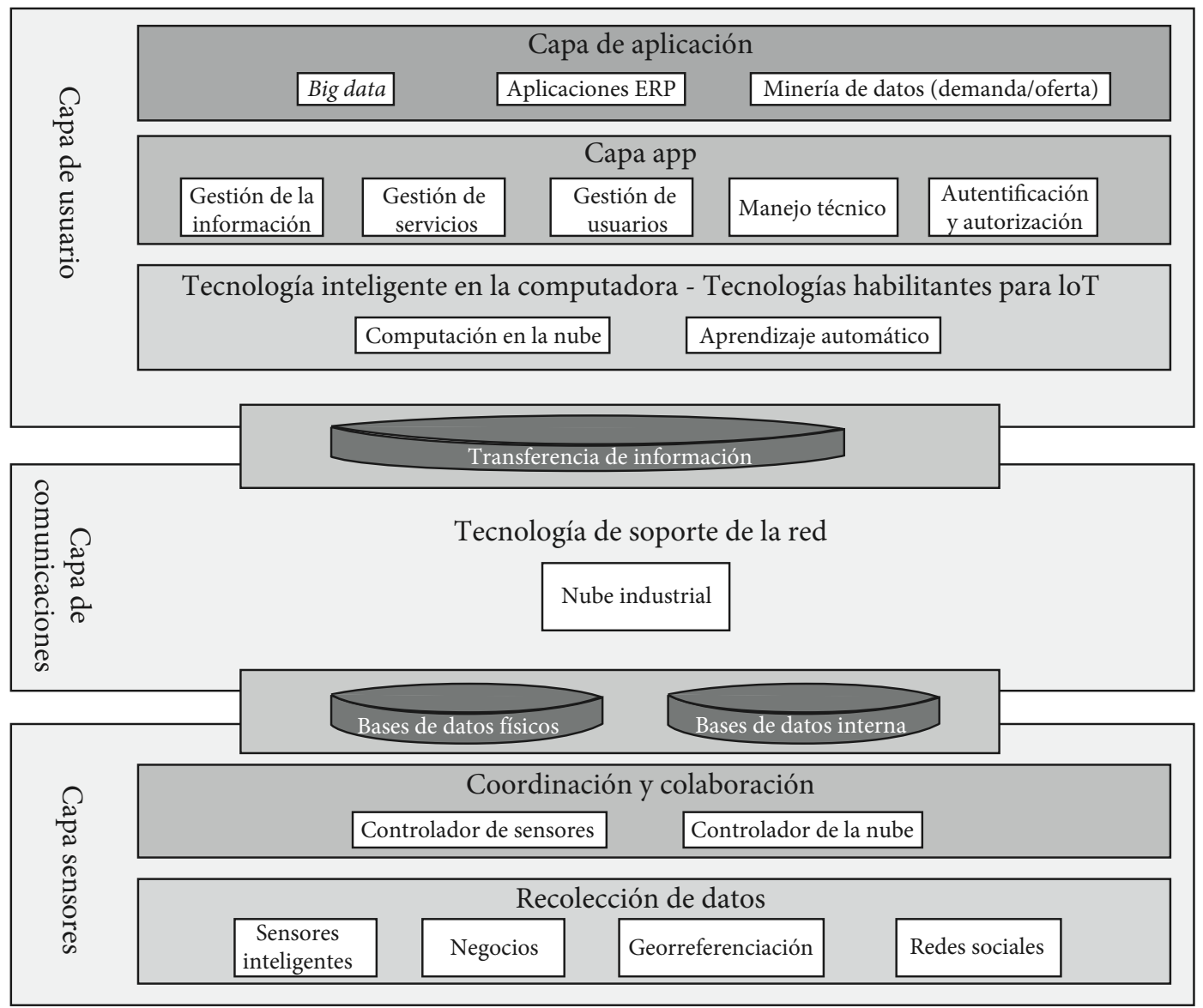

Figura 2. Propuesta de arquitectura en el contexto de la I4 Fuente: adaptado de [32] 


\subsubsection{Sensores inteligentes}

Los sensores inteligentes son dispositivos que convierten parámetros físicos en señales eléctricas y devuelven datos analógicos o digitales [37]. En otras palabras, los sensores permiten medir un parámetro físico concreto, o detectar un cambio en el entorno cercano a los mismos [38]. Se complementan con los actuadores, que se encargan de convertir las órdenes en acciones. Se emplearon los siguientes sensores en los eslabones de la cadena de suministro:

- Identificación por radiofrecuencia (RFID): esta tecnología consiste en una etiqueta RFID, que contiene la información de identificación única del producto, un lector que recoge la información almacenada en la etiqueta y un sistema servidor que almacena los datos [39]. Este tipo de sensores facilitó la identificación y administración de los inventarios, el monitoreo de los activos de la empresa, la detección de posición del producto dentro de la cadena de suministro (trazabilidad), el control de máquinas (diagnóstico y seguimiento) y controles de acceso por proximidad.

- Termopares: estos sensores se usaron para medir temperaturas; son usuales debido a su bajo costo, amplio rango de temperaturas y sus conexiones estándar.

- Sensor de desplazamiento láser: permitió realizar una medición exacta sin contacto alguno con el producto; la luz del láser cambia de intensidad dependiendo de las medidas del objeto.

- Sensores fotoeléctricos de barrera: se utilizaron para controlar las medidas especificadas de los productos.

- Celdas de carga: fue posible tener un control del peso de los productos por medio de estos sensores, que transmiten la información para ser controlada mediante un indicador de peso.

\subsubsection{Negocios}

Dentro de la cadena de suministro, la red de comunicación se realizó entre varias empresas, fábricas, proveedores y clientes. Cada sección optimizó su configuración en tiempo real en función de las exigencias y el estado de las secciones asociadas a la red; también creó el máximo beneficio para todos los eslabones con el intercambio de recursos limitados [3].

\subsubsection{Georreferenciación}

La distribución de materias primas y productos terminados es un elemento crucial en la cadena de suministro. La misión de la distribución es hacer llegar los productos correctos al lugar indicado en el momento preciso y con las condiciones requeridas.

En la recolección de datos de distribución como parte de la propuesta de arquitectura se utilizaron los siguientes elementos, que permitieron llevar un control en la logística:

- Sistema de información geográfica: con esta herramienta se obtuvo información sobre las características de los lugares, las condiciones de las rutas y el cálculo de rutas óptimas.

- Sistema de posicionamiento global: es un servicio gratuito y gran aliado en la logística, dado que permite a sus usuarios determinar con exactitud su ubicación y desplazarse a otro lugar. En la arquitectura propuesta se utilizó como un apoyo de navegación terrestre.

\subsubsection{Redes sociales}

El incremento del uso del internet fomentó el uso de las redes sociales, que son conexiones entre diferentes actores. Durante los últimos años, las técnicas de análisis para las redes sociales han adquirido importancia en la aplicación y uso de la minería de datos, brindando a las organizaciones una herramienta para la predicción de ventas y segmentación del mercado. A través de las técnicas API rest y streaming API [40], se extrajeron los datos de las redes sociales.

\subsubsection{Controlador de la nube}

Un controlador de nube es un dispositivo de almacenamiento que transfiere automáticamente los datos del almacenamiento local al almacenamiento en la nube. En el desarrollo de la arquitectura propuesta se empleó el servicio Amazon Web Services (Aws) de controladores de la nube. 


\subsubsection{Controlador de sensores}

Estos son dispositivos que almacenan y ejecutan los programas de control de los sensores y actuadores. Se utilizaron los controladores Autonics Sensors Controllers (ASC), que son compatibles con una amplia gama de sensores.

\subsection{Bases de datos}

Toda la información recolectada debe almacenarse; para esto se utilizan las bases de datos en las que se puede acceder a la información de manera segura y ágil. En la arquitectura propuesta se utilizaron dos tipos de bases de datos: una física y la otra interna.

- Bases de datos físicas: los datos se almacenan en el servidor de la empresa. Desde este servidor se suben los datos a la nube, lo que significa que la información se modifica en tiempo real.

- Bases de datos internas: se realizó un backup de la información para tener acceso a esta sin necesidad de tener conexión a Internet. Solo se realizan consultas sobre los datos ya existentes para el análisis y toma de decisiones.

\subsection{Capa de comunicaciones}

En esta capa se encuentra la infraestructura que almacena en una nube industrial. Acá es donde se da la integración entre las capas que recibe los datos y suministra información a las aplicaciones externas.

\subsubsection{Nube industrial}

Con el fin de acceder a la información de la cadena de suministro desde cualquier lugar y en tiempo real, es necesario tener conexión a Internet y acceso al servidor Aws donde se almacena la información, para descentralizarla y ponerla a disposición de quien la requiera.

\subsection{Transferencia de información}

Para el desarrollo de esta arquitectura, se utilizaron las TIC más comunes aplicadas a la cadena de suministro, Electronic Document Interchange (EDI) y Vendor Managed Inventory (VMI) con el fin de evitar fugas de información y pérdidas a lo largo del hilo digital. Para que la información esté disponible en un punto específico, es crucial compartirla en toda la cadena en tiempo real. Por lo tanto, se integraron fuentes dispares de datos de diferentes aplicaciones para crear una visión holística del proceso de extremo a extremo. Además, esta integración de datos incluye la información de los proveedores y clientes. Esta información es relevante para la adaptación de los procesos de fabricación [41].

\subsection{Capa de usuario}

Esta capa muestra por medio de una aplicación externa el monitoreo de materias primas, las fallas de equipos, el control de calidad, la programación de la producción, la predicción de demanda y las rutas óptimas de distribución, entre otros, con la ayuda de la computación en la nube, big data y el aprendizaje automático.

\subsubsection{Computación en la nube}

Se contrataron los servicios de Amazon Machine Learning, que permiten acceder a los servicios de software y almacenamiento desde cualquier lugar por medio de Internet.

\subsubsection{Aprendizaje automático}

El aprendizaje automático para esta arquitectura se soportó en diferentes herramientas, como Hadoop, Flink y Kafka; también puede ser soportado en otras técnicas desarrolladas para la organización que lo requiera.

\subsubsection{Capa de app}

Para ingresar a la app desarrollada se accede mediante una cuenta de usuario, autentificando y autorizando el ingreso para asegurar la información que esta contiene, y administrando de esta manera los usuarios que pueden tener acceso a la aplicación. A través de la $a p p$, se puede gestionar la información durante su ciclo de vida (desde su captura hasta su eliminación). La aplicación cuenta también con un módulo de soporte técnico por si se requiere.

\subsubsection{Big data}

Por medio del big data se analizaron, seleccionaron y procesaron los datos recolectados de los elementos anteriormente descritos en la capa de sensores. Para 
el desarrollo de esta arquitectura, se utilizó Apache Storm, el cual es un sistema de computación distribuida en tiempo real, orientado a procesar flujos constantes de datos, como datos de sensores.

\subsubsection{Minería de datos en la oferta y demanda}

La exploración de datos de la oferta de materias primas y de la demanda de productos terminados brinda una comprensión de los patrones y tendencias de estos datos para así ser procesados por medio del big data. Para esta arquitectura se utilizó Apache Storm, que utiliza técnicas predictivas como métodos bayesianos y algoritmos genéticos.

\subsubsection{Aplicaciones ERP}

Los sistemas ERP se usaron para la planificación de recursos empresariales desde la plataforma SAP. Típicamente manejan la producción, la logística, la distribución, el inventario, los envíos, las facturas y contabilidad de la compañía de forma modular. La aplicación desarrollada para esta propuesta de arquitectura cuenta con cinco módulos: a) pronóstico de demanda, b) reporte de fallas, c) reporte de producción, d) reporte de proveedores, y e) ruteo.

\subsection{Validación de la propuesta}

La validación de la interconexión entre las capas de sensores y la de aplicación se realizó con una prueba a la fase de usuario, en la cual se evalúo la app final que se muestra en la figura 3, llamada ERP for I4, aplicando el "Modelo para la medición de usabilidad de aplicaciones móviles, mediante el análisis de atributos y métodos de evaluación de Usabilidad" [42], que da los lineamientos en número de usuarios, expertos, atributos y subatributos.

Al aplicar el modelo, se necesitó de cuatro expertos con experiencia en el software SAP y la modificación de sus módulos; asimismo, eran ingenieros de producción/logística y con experiencia profesional en el cargo de jefe de producción. Se tomaron cinco usuarios que fueron aquellos empleados que tenían que ver con la cadena logística de la organización, además de establecer los atributos, subatributos y heurísticas que se muestran a continuación:

Atributos: a) el usuario entiende la funcionalidad de la $a p p$, b la app propuesta ayuda al usuario a interactuar con los eslabones de la cadena de suministro, c) la $a p p$ funciona de manera ágil, d) la $a p p$ maneja información en tiempo real, y e) la información que brinda la app es de ayuda para los eslabones de la cadena de suministro.
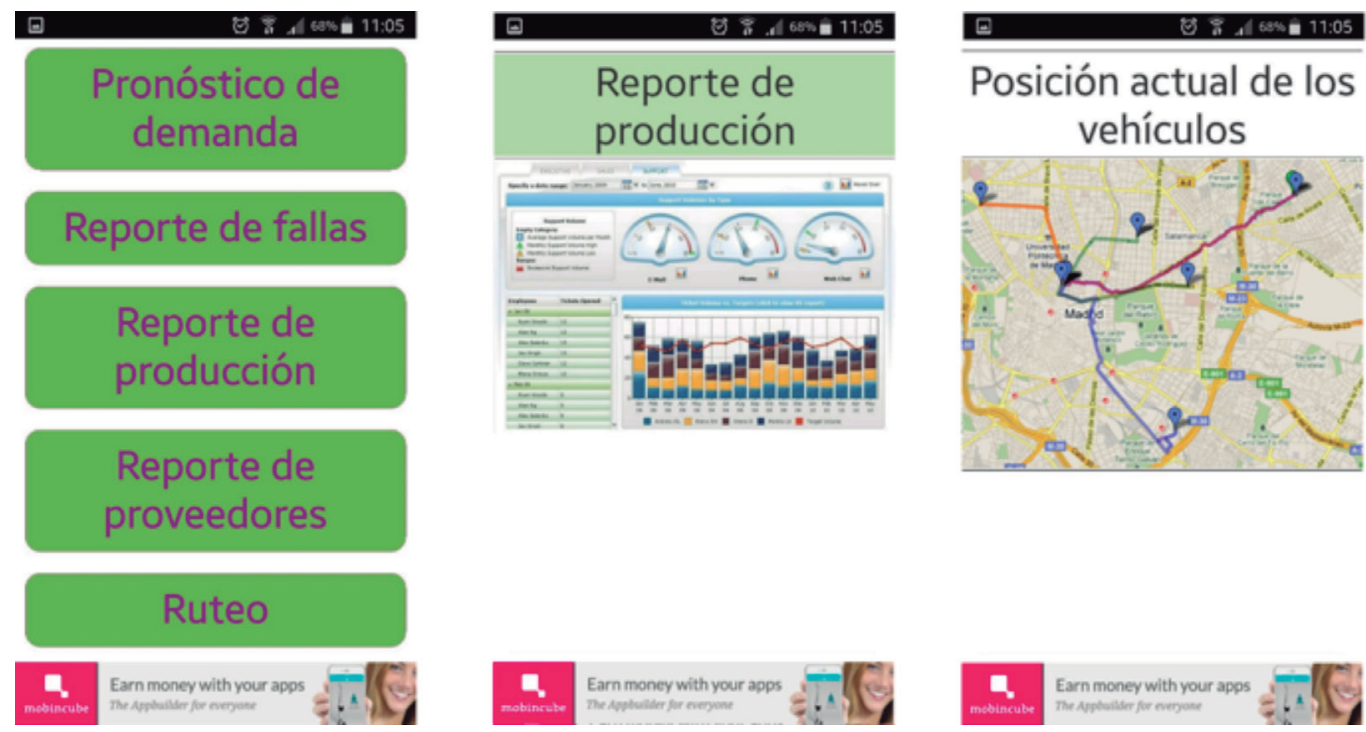

Figura 3. Interfaz de aplicación de usuario basada en la arquitectura propuesta Fuente: elaboración propia 
Subatributos: a) es de fácil comprensión la app, b) es fácil manejar la $a p p$ y c) la información obtenida es fácil de analizar.

Heurísticas: a) la app propuesta ofrece una manera rápida de obtener información de la cadena de suministro, b) las empresas pueden beneficiarse de la $a p p$ y c) el Internet de las cosas puede beneficiarse de la $a p p$.

Una vez aplicado el modelo con las propiedades anteriormente descritas, se obtuvo como resultado una usabilidad de la aplicación del 91\%, como se muestra en la tabla 1 .

Tabla 1. Resultados de usabilidad de la aplicación móvil: ERP for I4

\begin{tabular}{|c|c|}
\hline Atributo & Puntaje \\
\hline Atributo a & $95 \%$ \\
\hline Atributo b & $88 \%$ \\
\hline Atributo c & $94 \%$ \\
\hline Atributo d & $91 \%$ \\
\hline Atributo e & $87 \%$ \\
\hline Usabilidad & $91 \%$ \\
\hline
\end{tabular}

Fuente: elaboración propia

Con los datos obtenidos del modelo en la aplicación móvil, se muestra que la aplicación ERP for I4 cumple con los parámetros necesarios para dar validez a la arquitectura propuesta, en la cual la cadena de suministro se integre a la I4 de manera exitosa.

\section{Discusión}

El modelo de usabilidad de aplicaciones móviles que se utilizó para evaluar la aplicación móvil ERP for I4 arrojó un resultado de usabilidad del 91\%, por lo cual se puede afirmar que la arquitectura propuesta cumple su función a la hora de integrar los eslabones de la cadena de suministro con la I4, específicamente, la capa de sensores junto con la capa de aplicación. A partir de la revisión del estado del arte y el desarrollo de la arquitectura, se evidenciaron los siguientes resultados y discusiones.

\subsection{Integración}

La fabricación digital implica que las plataformas integradas de planificación y ejecución conectan todas las partes de la cadena de valor $[43,44]$, lo que consigue que en cualquier punto de la cadena de suministro se obtuviera información al instante para ser usada en la toma de decisiones acertadas $[45,46]$.

\subsection{Costo}

La información incorrecta y las decisiones erróneas tomadas dentro de la cadena de suministro generan pérdidas económicas [39, 47]. Esto se disminuyó con la ayuda de la app ERP for I4, pues esta presentaba datos precisos en todos los procesos de la organización en el contexto adecuado y el formato necesario para tomar decisiones acertadas. Adicionalmente, la cooperación entre los eslabones de la cadena de suministro redujo costos al tener una completa comunicación en tiempo real. Sin embargo, para implementar las soluciones de la I4, se requieren altas inversiones para crear una infraestructura de red robusta y segura, y actualizar o reemplazar sistemas de la organización [40]. La justificación de estas inversiones requiere que los beneficios se cuantifiquen de manera inequívoca y fiable.

\subsection{Flexibilidad}

Se crearon sistemas flexibles listos para el cambio [3] y listos para nuevas oportunidades al optimizar los procesos basándose en el análisis de datos. Cada vez que el aprendizaje automático ofrece mayor predicción en los procesos [44], el sistema se renueva a partir de los datos que arroja el modelo de predicción.

\subsection{Tiempo}

Las máquinas inteligentes comparten información en tiempo real [7] continuamente sobre los niveles de stock, problemas, errores o los cambios en pedidos. Los procesos y los plazos se controlan para aumentar la eficiencia y optimizar los plazos de entrega [37, 41]. Se obtuvo una disminución de tiempo en los procesos debido a los datos recolectados por los sensores, con lo cual fue más fácil predecir las decisiones a tomar que generan valor al proceso, eliminan los tiempos muertos y se realizó un plan de mantenimiento adecuado. En el ámbito de la logística, también se disminuyó el tiempo de transporte de mercancías al elegirse la ruta óptima y el proveedor que tenga los menores tiempos de envío. 


\subsection{Fábrica inteligente}

$\mathrm{Al}$ integrar la cadena de suministro en la I4, se crean fábricas inteligentes en las cuales no solo todos los recursos de fabricación (sensores, actuadores, máquinas, robots, transportadores, entre otros) están conectados e intercambiando información de forma automática [3], [7], sino que la fábrica es lo suficientemente inteligente como para proporcionar procesos de producción flexibles y adaptativos que resuelven los problemas que surjan en una instalación de producción [46]. Esto se vio a partir de la app. Las fábricas obtuvieron información en tiempo real, lo que permitió tomar el control sobre cada proceso de la empresa.

\subsection{Canales de distribución}

Se usaron transportes inteligentes con seguimiento en tiempo real del producto, utilizando sistemas de georreferenciación conectados a la nube [4]. Adicionalmente, se instalaron sensores en los vehículos para detectar daños y averías, y un sistema de transformación de datos de tráfico para la distribución correcta de los elementos a través de la cadena de suministro [45], [48].

\subsection{Máquinas inteligentes}

La comunicación máquina-máquina es esencial en los sistemas cibernéticos, ya que permite que los dispositivos conectados a la red inicien y accionen la comunicación sin intervención ni ayuda humana [7], [37]. Las máquinas que trabajan en una línea de producción se suministraron información del proceso que realizaban; esto fue un gran aliado para detectar errores en la línea de producción.

\subsection{Producto inteligente}

Se trata de un nuevo tipo de producto generado en la industria, que recopila datos de proceso para el análisis durante y después de su producción [49], [50]. Estos productos están integrados con sensores, componentes identificables y procesadores que llevan la información [3]; esto permite a los objetos inteligentes comunicarse con otros objetos, procesar datos de entorno y realizar eventos [38]. Los productos utilizados en la aplicación estaban identificados con etiquetas RTIF, que permitieron transferir información de cada producto respecto a su posición en la cadena suministro (trazabilidad).

\subsection{Impacto social}

Al tener un control total de los inventarios, se mostró que solo se necesita un empleado en este proceso. El uso de la tecnología en algunos procesos hace que se pueda prescindir de empleados que realizaban estas labores, por lo que se recomienda que estos sean capacitados en temas de la I4 para que brinden nuevos conocimientos a la organización. [45] recomienda determinar y documentar el impacto sobre el trabajo y el empleo (oportunidades y riesgos) junto con las acciones necesarias para lograr políticas laborales y de formación orientadas hacia los trabajadores.

\section{Conclusión}

Como resultado de la investigación presentada, se puede concluir que existe una relación positiva entre la arquitectura propuesta y la eficiencia de la cadena de suministro, debido a dos factores principales: el primero está relacionado con interacción en tiempo real entre los eslabones de la cadena de suministro apoyado en la computación en la nube. El segundo factor es el aprendizaje automático que permite la toma de decisiones óptimas y eficientes.

Los esfuerzos que hacen las empresas para crear ventajas competitivas pueden reforzarse debido a la inmersión en la I4; sin embargo, las organizaciones deben tener en cuenta que la inclusión en la nueva revolución industrial trae consigo cambios en las tecnologías que se utilizan para la recolección de datos, el análisis y su transformación, y estos cambios de tecnología representan una inversión que deben hacer las organizaciones.

Luego de la validación de la propuesta, se pudo determinar que el uso de la aplicación móvil desarrollada brinda una iniciativa a las organizaciones para hacer parte de la I4 debido a que la interfaz presentada es adecuada para la revisión de la información deseada desde cualquier punto de la cadena de suministro en tiempo real.

\section{Futuras líneas de investigación}

Para futuras investigaciones, se debe tener en cuenta la seguridad de la información por medio de encriptaciones que permitan la confidencialidad de los datos y la protección de estos frente a 
terceros. Se deben establecer protocolos de seguridad para el acceso a la información.

Se deben estandarizar las tecnologías para permitir la integración de todos los elementos por medios de diferentes plataformas, independiente del software o hardware con los que cuenten, estudiando los enfoques y mecanismos para apoyar la interoperabilidad entre los componentes.

Adicionalmente, el desarrollo de diversos algoritmos para el procesamiento de datos procedentes de la cadena de suministro para proporcionar una mejor comprensión del funcionamiento de la cadena de suministro global.

\section{Referencias}

[1] J. Ang, C. Goh, A. Saldivar y Y. Li, "Energy-Efficient Through-Life Smart Design, Manufacturing and Operation of Ships in an Industry 4.0 Environment", Energies, vol. 10, n. ${ }^{\circ}$ 5, p. 610, 2017. [Online]. doi: https://doi.org/10.3390/en10050610

[2] B. Mrugalska y M. K. Wyrwicka, "Towards Lean Production in Industry 4.0", Procedia Eng., vol. 182, pp. 466-473, 2017. [Online]. doi: https://doi. org/10.1016/j.proeng.2017.03.135

[3] J. Qin, Y. Liu y R. Grosvenor, "A Categorical Framework of Manufacturing for Industry 4.0 and beyond", Procedia CIRP, vol. 52, pp. 173-8, 2016. [Online]. doi: https://doi.org/10.1016/j.procir.2016.08.005

[4] A. Jayaram, "Lean Six Sigma Approach for Global Supply Chain Management Using Industry 4.0 and IIoT", 2016 2nd Int. Conf. Contemp. Comput. Informatics, pp. 89-94, 2016. [Online]. doi: https://doi. org/10.1109/IC3I.2016.7917940

[5] International Electrotechnical Commission, "Factory of the future", White Pap. Futur. Fact., pp. 44-7, 2015. [Online]. Disponible en http://www.qualitymag.com/articles/93484-stepping-up-to-the-factory-of-the-future

[6] V. Díez, A. Arriola, I. Val y M. Vélez, "Validation of RF Communication Systems for Industry 4. 0 . through Channel Modeling and Emulation", IEEE Int. Work, pp. 2-7, 2017. [Online]. doi: https://doi. org/10.1109/ECMSM.2017.7945906

[7] F. Shrouf, J. Ordieres y G. Miragliotta, "Smart Factories in Industry 4.0: A Review of the Concept and of Energy Management Approached in Production Based on the Internet of Things Paradigm", IEEE International Conference on Industrial Engineering and Engineering Management, 2014, vol. 2015, pp. 697-701, Mar. 2015. [Online]. doi: https://doi. org/10.1109/IEEM.2014.7058728

[8] L. Spendla, M. Kebisek, P. Tanuska y L. Hrcka, "ConceptofPredictiveMaintenanceofProductionSystems in Accordance with Industry 4.0", SAMI 2017 IEEE 15th Int. Symp. Appl. Mach. Intell. Informatics, Proc., pp. 405-10, 2017. [Online]. doi: https://doi. org/10.1109/SAMI.2017.7880343

[9] H. Meissner, R. Ilsen y J. C. Aurich, "Analysis of Control Architectures in the Context of Industry 4.0", Procedia CIRP, vol. 62, pp. 165-9, 2017. [Online]. doi: https://doi.org/10.1016/j.procir.2016.06.113

[10] L. Thames and D. Schaefer, "Software-defined Cloud Manufacturing for Industry 4.0", Procedia CIRP, vol. 52, pp. 12-17, 2016. [Online]. doi: https://doi. org/10.1016/j.procir.2016.07.041

[11] M. K. Soundarapandian, K. Suri, J. Cadavid, I. Barosan, M. Van Den Brand, M. Alferez y S. Gerard, "Towards Industry 4.0: Gap Analysis between Current Automotive MES and Industry Standards using Model-Based Requirement Engineering", 2017 IEEE International Conference on Software Architecture Workshops, pp. 29-35, 2017. [Online]. Disponible en http://ieeexplore.ieee.org/document/7958432/

[12] A. Chaouni Benabdellah, A. Benghabrit, I. Bouhaddou y E. M. Zemmouri, "Big data for Supply Chain Management: Opportunities and Challenges", Int. J. Sci. Eng. Res., vol. 7, n. ${ }^{\circ} 11$, pp. 20-5. [Online]. doi: https://doi.org/10.1016/j.cie.2016.07.013

[13] D. Ghosh, "Big data in Logistics and Supply Chain Management - A Rethinking Step", Int. Symp. Adv. Comput. Commun., pp. 168-173, 2015. [Online]. doi: https://doi.org/10.1109/ISACC.2015.7377336

[14] M. J. Farooq and Q. Zhu, "Secure and Reconfigurable Network Design for Critical Information Dissemination in the Internet of Battlefield Things (IoBT)", 15th International Symposium on Modeling and Optimization in Mobile, Ad Hoc y Wireless Networks (WiOpt), 2017, pp. 1-8. [Online]. doi: https:// doi.org/10.23919/WIOPT.2017.7959892

[15] F. Alkhabbas, R. Spalazzese y P. Davidsson, "Emergent Configurations in the Internet of Things as System of Systems", Proc. Jt. 5th Int. Work. Softw. Eng. Syst. 11th Work. Distrib. Softw. Dev. Softw. Ecosyst. Syst., n. ${ }^{\circ}$ iii, pp. 70-1, 2017. [Online]. doi: https://doi. org/10.1109/JSOS.2017.6

[16] A. Iglesias-Urkia, Markel; Orive, Adrián; Urbieta "Analysis of CoAP Implementations for Industrial Internet of Things: A Survey", 8th Int. Conf. Ambient Syst. Networks Technol. (ANT 2017), vol. 0, n. ${ }^{\circ}$ 2016, 2017. [Online]. doi: https://doi.org/10.1016/j. procs.2017.05.323 
[17] K. Rose, S. Eldridge y C. Lyman, “The Internet of Things: An Overview", Internet Soc., n. ${ }^{\circ}$ October, p. 53, 2015. [Online]. Disponible en https://cdn.prod. internetsociety.org/wp-content/uploads/2017/08/ ISOC-IoT-Overview-20151221-en.pdf

[18] T. Saarikko, U. H. Westergren y T. Blomquist, “The Internet of Things: Are You Ready for What's Coming?", Bus. Horiz., 2017. [Online]. doi: https://doi. org/10.1016/j.bushor.2017.05.010

[19] D. Mourtzis, E. Vlachou y N. Milas, "Industrial Big Data as a Result of Iот Adoption in Manufacturing", Procedia CIRP, vol. 55, pp. 290-5, 2016. [Online]. doi: https://doi.org/10.1016/j.procir.2016.07.038

[20] F. Nie, "A Value-oriented Model of Big Data-Driven Knowledge Management and Collaborative Services: A Meteorological Service Case”, pp. 511-6, 2017. doi: https://doi.org/10.1109/INFOMAN.2017.7950438

[21] C. Tidbits, "Cloud Computing Changes Data Integration Forever: What's Needed Right Now", IEEE Comput. Soc., pp. 50-3, 2017. [Online]. doi: https:// doi.org/10.1109/MCC.2017.47

[22] N. Khan and A. Al-Yasiri, "Identifying Cloud Security Threats to Strengthen Cloud Computing Adoption Framework", Procedia Comput. Sci., vol. 94, pp. 485-90, 2016. [Online]. doi: https://doi.org/10.1016/j.procs.2016.08.075

[23] M. H. Raza, A. F. Adenola, A. Nafarieh y W. Robertson, "The Slow Adoption of Cloud Computing and IT Workforce", Procedia Comput. Sci., vol. 52, n. ${ }^{\circ}$ 1, pp. 1114-9, 2015. [Online]. doi: https://doi. org/10.1016/j.procs.2015.05.128

[24] T.-M. Choi y B. Shen, "A System of Systems Framework for Sustainable Fashion Supply Chain Management in the Big Data Era", 2016 IEEE 14Th Int. Conf. Ind. Informatics, pp. 902-8, 2016. [Online]. doi: https://doi.org/10.1109/INDIN.2016.7819290

[25] S. A. Hussain, M. Fatima, A. Saeed, I. Raza y R. K. Shahzad, "Multilevel classification of security concerns in cloud computing", Appl. Comput. Informatics, vol. 13, n. ${ }^{\circ} 1$, pp. 57-65, 2017. [Online]. doi: https://doi.org/10.1016/j.aci.2016.03.001

[26] D. Pop, "Machine Learning and Cloud Computing: Survey of Distributed and SaaS Solutions", Inst. e-Austria Timisoara, Tech. Rep 1, 2012. [Online]. Disponible en https://arxiv.org/pdf/1603.08767.pdf

[27] K. Christensen, S. Nørskov, L. Frederiksen y J. Scholderer, "In Search of New Product Ideas: Identifying Ideas in Online Communities by Machine Learning and Text Mining", Creat. Innov. Manag., vol. 26, n. ${ }^{\circ}$ 1, pp. 17-30, 2017. [Online]. doi: https://doi. org/10.1111/caim.12202

[28] A. Talwar y Y. Kumar, "Machine Learning: An Artificial Intelligence Methodology”, Int. J. Eng. Comput.
Sci., vol. 2, n. ${ }^{\circ} 12$, pp. 3400-3405, 2013. [Online]. Disponible en http://www.ijecs.in/issue/v2-i12/11\%20 ijecs.pdf

[29] J. Di Deco-Sampedro y J. Díaz-García, "Estudio y aplicación de técnicas de aprendizaje automático orientadas al ámbito médico: estimación y explicación de predicciones individuales", p. 103, 2012. [Online]. Disponible en https://repositorio.uam.es/ handle/10486/12100

[30] A. Stăncioiu, “The Fourth Industrial Revolution”, n. 1, pp. 74-9, 2017. [Online]. Disponible en http://bit. ly/2wncneN

[31] H. Lasi, P. Fettke, H.-G. Kemper, T. Feld y M. Hoffmann, "Industry 4.0", Bus. Inf. Syst. Eng., vol. 6, n. ${ }^{\circ}$ 4, p. 239, 2014. [Online]. Disponible en https:// www.cgi.com/en/white-paper/Industry-4-making-your-business-more-competitive

[32] J. Manuel, C. Lovelle, C. Enrique y M. Marín, "Metamodelo para la integración de la Internet de las cosas y redes sociales", 2014. [Online]. Disponible en http://di002.edv.uniovi.es/ cueva/investigacion/ tesis/Tesis-JoseIgnacio.pdf

[33] A. Arvind and D. Bourne, "Architecture for Industry 4.0-based Manufacturing Systems", n. ${ }^{\circ}$ July, 2016. [Online]. Disponible en http://ri.cmu.edu/publications/architecture-for-industry-4-0-based-manufacturing-systems/

[34] M. A. Pisching, F. Junqueira, D. J. dos Santos-Filho y P. E. Miyagi, "An Architecture for Organizing and Locating Services To the Industry 4.0", January, pp. 0-8, 2015. [Online]. doi: https://doi.org/10.20906/ CPS/COB-2015-0415

[35] M. Hermann, T. Pentek y B. Otto, "Design Principles for Industrie 4.0 Scenarios", 2016 49th Hawaii Int. Conf. Syst. Sci., IEEE, 2016: pp. 3928-3937, 2016. [Online]. doi: http://doi.ieeecomputersociety. org/10.1109/HICSS.2016.488

[36] J. H. Ang, C. Goh y Y. Li, "Smart Design for Ships in a Smart Product Through-Life and Industry 4.0 Environment", 2016 IEEE Congr. Evol. Comput. CEC 2016, pp. 5301-8, 2016. [Online]. doi: https://doi. org/10.1109/CEC.2016.7748364

[37] X. Liu y O. Baiocchi, "A Comparison of the Definitions for Smart Sensors, Smart Objects and Things in IOT", 7th IEEE Annu. Inf. Technol. Electron. Mob. Commun. Conf. IEEE IEMCON 2016, pp. 1-4, 2016. [Online]. doi: https://doi.org/10.1109/IEMCON.2016.7746311

[38] C. González, D. Meana-Llorián, C. Pelayo G-Bustelo y J. M. Cueva-Lovelle, "A Review about Smart Objects, Sensors, and Actuators", Int. J. Interact. Multimed. Artif. Intell, pp. 7-10, 2017. [Online]. doi: https://doi.org/10.9781/ijimai.2017.431 
[39] A. M. Turri, R. J. Smith y S. W. Kopp, "Privacy and RFID Technology: A Review of Regulatory Efforts”, J. Consum. Aff., vol. 51, n. ${ }^{\circ}$ 2, pp. 329-54, 2017. [Online]. doi: https://doi.org/10.1111/joca.12133

[40] E. Asensio-Blasco, "Aplicación de técnicas de minería de datos en redes sociales/web", p. 50, 2015. [Online]. Disponible en https://riunet.upv.es/handle/10251/56102

[41] D. Wee, R. Kelly, J. Cattel y M. Breunig, "Industry 4.0: How to Navigate Digitization of the Manufacturing Sector", McKinsey Co., pp. 1-62, 2015. [Online]. Disponible en https://www.mckinsey.de/files/ mck_industry_40_report.pdf

[42] J. I. Rodríguez-Molano, E. Sierra-Yara y L. K. Jaramillo-García, "Model for Measuring Usability of Survey Mobile Apps, by Analysis of Usability Evaluation Methods and Attributes", 2015 10th Iber. Conf. Inf. Syst. Technol. Cist. 2015, 2015. [Online]. doi: https://doi.org/10.1109/CISTI.2015.7170420

[43] M. Gubán y G. Kovács, "Industry 4.0 Conception”, Bull. Eng., vol. X, n. ${ }^{\circ}$ 1, pp. 111-4, 2017. [Online]. Disponible en https://search.proquest.com/openview/ d6ee02cbb198937e93f6421f4f692c76/1?pq-origsite $=$ gscholar $\& \mathrm{cbl}=616471$

[44] T. Fukui, "A Systems Approach to big data Technology Applied to Supply Chain”, pp. 3732-6, 2016. [Online]. doi: https://doi.org/10.1109/BigData.2016. 7841041

[45] R. Rana, M. Staron, J. Hansson, M. Nilsson y W. Meding, "A Framework for Adoption of Machine Learning in Industry for Software Defect Prediction", Proc. 9th Int. Conf. Softw. Eng. Appl., pp. 383-
392, 2014. [Online]. Disponible en http://ieeexplore. ieee.org/abstract/document/7293887/

[46] H. Kagermann, W. Wahlster y J. Helbig, Recommendations for Implementing the Strategic Initiative INDUSTRIE 4.0, Frankfurt: National Academy of Science and Engineering, April 2013, p. 82. [Online]. Disponible en http://www.acatech.de/fileadmin/user_upload/ Baumstruktur_nach_Website/Acatech/root/de/Material_fuer_Sonderseiten/Industrie_4.0/Final_report_Industrie_4.0_accessible.pdf

[47] A. H. Glas y F. C. Kleemann, “The Impact of Industry 4.0 on Procurement and Supply Management: A Conceptual and Qualitative Analysis", Int. J. Bus. Manag. Invent. vol. 5, n. ${ }^{\circ}$ 6, pp. 55-66, 2016. [Online]. Disponible en http://www.ijbmi.org/papers/ Vol(5)6/I0506055066.pdf

[48] J. Wang, R. R. Muddada, H. Wang, J. Ding, Y. Lin, C. Liu y W. Zhang, "Toward a Resilient Holistic Supply Chain Network System: Concept, Review and Future Direction", IEEE Syst. J., vol. 10, n. ${ }^{\circ} 2$, pp. 410-21, 2016. [Online]. doi: https://doi.org/10.1109/ JSYST.2014.2363161

[49] D. Kolberg y D. Zühlke, "Lean Automation Enabled by Industry 4.0 Technologies", IFAC-Papers On Line, vol. 28, n. ${ }^{\circ} 3$, pp. 1870-1875, 2015. [Online]. doi: https://doi.org/10.1016/j.ifacol.2015.06.359

[50] M. E. Porter y J. E. Heppelmann, "How Smart, Connected Products are Transforming Competition", Harvard Business Review, vol. 92, n. ${ }^{\circ} 11$, pp. 64-88, 2014. [Online]. Disponible en http://www.gospi. fr/IMG/pdf/porter-2014-hbr_how-smart-connected-products-are-transforming-competitionhbr-2014.pdf 